\title{
UN ESTUDIO DEL RENDIMIENTO DIFERENCIAL EN EL RECONOCIMIENTO DE EMOCIONES SIMPLES Y CONGNITIVAS ENTRE AUTISTAS, DEFCIENTES MENTALES Y POBLACIÓN GENERAL
}

\author{
DOMINGO GARCÍA-VILLAMISAR y AQUILINO POLAINO-LORENTE \\ Universidad Complutense de Madrid
}

(Recibido el 11 de mayo de 1998)

\begin{abstract}
Grupos de autistas $(n=17)$ y no autistas adultos con retraso mental $(n=17)$, agrupados individualmente por EC y EM y sexo, junto con sujetos de la población general (n = 17), igualados por EC fueron examinados en su habilidad para reconocer la expresión facial de emociones. Los autistas demostraron una leve alteración en su apreciación de las expresiones emocionales. Reconocen por igual tanto las emociones simples como las emociones cognitivas. Sin embargo, emplearon más tiempo en el reconocimiento de las emociones cognitivas.
\end{abstract}

Palabras clave: Emociones simples y cognitivas, reconocimiento de emociones, adultos autistas.

\section{A differential study of the recognition of simple and cognitive emotions by autistic mentally retarded and normal adults}

Groups of autistic $(n=17)$ and non autistic retarded young adults $(n=17)$, individually matched for CA, verbal MA and sex, together with a group of normal young $(n=17)$, matched for CA and sex, were tested for their ability to recognice facial expressions of emotions. The autistic subjects were relatively impaired in appreciation of emotional expressions. They recognize equal the basic and cognitive emotions. However, they use more time in perception of the cognitive emotions.

Key words: Autism, Cognitive and basic emotions, Perception, Adults with autism.

\section{INTRODUCCIÓN}

Desde que el autismo se concibió como un trastorno profundo del desarrollo, los especialistas han puesto desde siempre de manifiesto la incapacidad de los pacientes autistas para relacionarse con la gente, lo que constituye uno de sus síntomas patognomónicos (Kanner, 1943).

\footnotetext{
Agradecimientos: Los autores agradecen a Carmen Muela, Pedro Luis Nieto, Eva Yenes e Inmaculada Navarro su colaboración en la elaboración y aplicación de las pruebas de esta investigación. Reconocen y agradecen igualmente a la Asociación Nuevo Horizonte de las Rozas (Madrid) y al Centro de Educación Especial Virgen de Lourdes de Majadahonda de Madrid y a todo su cuerpo técnico las facilidades que ofrecieron para la realización de esta investigación. Eva Yenes ha colaborado también en el mecanografiado del texto. Al mismo tiempo, los autores quieren agradecer al Dr. Peter Hobson del Instituto de Psiquiatría de Londres las sugerencias y reco-
}

En la última década, diversas investigaciones llevadas a cabo por Hobson ( $C f r$. Hobson, 1993), han tratado de verificar la hipótesis de que los autistas están afectados por una incapacidad innata para conocer e interpretar las respuestas emocionales de los demás, en donde asentaría la causa fundamental de los déficits sociales que en ellos se observan.

mendaciones ofrecidas para el diseño y desarrollo de esta investigación. Igualmente agradecen a un revisor anónimo las sugerencias ofrecidas para la redacción final de este artículo. Esta investigación fue posible gracias a la ayuda económica concedida por la Universidad Complutense de Madrid para la realización de proyectos de investigación precompetitivos.

Correspondencia: Domingo García-Villamisar. Departamento de Personalidad, Evaluación y Psicologia Clrnica I, Despacho 3403, Facultad de Educación. Avda. de Juan XXIII, sn. 28040 Madrid. 
Los resultados de algunas investigaciones realizadas por nosotros ponen de manifiesto que los adultos autistas sufren un considerable déficit en la percepción y en el reconocimiento de emociones (García-Villamisar y Polaino-Lorente 1998 b y 1998c).

De otra parte, otras investigaciones sostienen que la causa de los déficits sociales en el autismo reside en que estos pacientes sufren un retraso en la adquisición de los elementos que se concitan en la así llamada "Teoría de la mente» (Cfr., Baron-Cohen, 1993; Happé, 1994).

Uno de los pioneros en el estudio de las atribuciones acerca de las emociones, Harris (1989), postula que los niños sanos, con edades comprendidas entre los 3 y los 4 años, conocen el hecho de que ciertas situaciones puedan provocar determinadas respuestas emocionales (Cfr., además, Borke, 1971; Trabasso et al., 1981; Baron-Cohen, 1991; BaronCohen et al., 1993). A esta edad, los niños son capaces de percibir cómo los deseos pueden llegar a disparar ciertas respuestas emocionales. (Wellman y Bartsch, 1988; Vellman y Woolley, 1990; Yuill, 1984; Baron-Cohen, 1991 y 1993; Baron-Cohen et al., 1993). Entre los $5 \mathrm{y}$ los 6 años, los niños son también capaces de identificar ciertas creencias como causa de las emociones (Harris, 1989; BaronCohen, 1991; Wellman, 1990; BaronCohen, 1991y 1993). En opinión de Harris (1989, 1993, 1994), las habilidades relativas a la «metarrepresentación» cognitiva juegan en los niños un papel determinante en lo que se refiere al complejo proceso de desarrollo de la comprensión de las emociones.

La metarrepresentación ha sido definida por Leslie (1988 y 1993) como un cierto tipo de representación interna, que determinaría la capacidad de la mente humana para caracterizar y manipular sus propias actitudes respecto de la información percibida. Los autores antes cita- dos sostienen que los déficits manifestados por los autistas en sus relaciones interpersonales, incluido el reconocimiento de las emociones, serían debidos a las dificultades que experimentan para procesar y/o formar metarrepresentaciones. En consecuencia con ello, el manifiesto déficit de los pacientes autistas para el procesamiento de las emociones (Hobson, 1993), sería la consecuencia derivada de una ausencia o disfunción en el mecanismo de desacoplamiento.

Esta capacidad de metarrepresentación, a la que se viene haciendo referencia, sería fundamental, según Harris $(1990,1993)$, para el reconocimiento y la comprensión de las emociones. Esto significa que, simular o fingir la realidad $y$, consecuentemente, imaginar y describir una realidad, tal y como otras personas supuestamente la perciben, se erige en la clave a través de la cual los niños se abren a la consideración del punto de vista adoptado por los otros. De esta suerte, a partir de un cierto nivel de desarrollo, es como los niños pueden llegar a entender la alegría, los miedos, la esperanza o las penas que se manifiestan en otras personas. El deficit de metarrepresentación es la causa de que los autistas carezcan de una teoría de la mente que les permita hacer conjeturas acerca de los estados mentales de los demás.

\section{Emociones simples vs. emociones cognitivas}

De acuerdo con Baron-Cohen (1993), Wellman (1990), Perner (1991), y otros numerosos autores, las emociones pueden clasificarse en torno a dos extremos fundamentales: las emociones simples vs. las emociones cognitivas.

Se asume que cualquier emoción puede ser etiquetada, por tanto, como simple o cognitiva. Todo dependerá de la atribución causal del observador. Supongamos, 
por ejemplo que "Alberto está muy contento, porque fue a un cumpleaños muy divertido"; este sería un ejemplo de emoción simple, puesto que la emoción (estar (contento) estaría causada por una situación del mundo real (un cumpleaños muy divertido). Por el contrario, en el ejemplo "Ana está muy contenta, porque piensa en su próximo cumpleaños», se trataría de una emoción cognitiva porque es una emoción causada por una creencia cognitiva (expectativa), cuyo contenido no tiene relación alguna con la realidad actual (Baron-Cohen, 1993).

Baron-Cohen (1993) sostiene que algunas emociones tales como la alegría, la tristeza, el enfado y el miedo son típicamente situacionales debido a que cuando observamos estas emociones tal como son expresadas por los demás, las atribuimos a causas concretas como situaciones divertidas, aburridas, etc. Sólo en el caso de que esta explicación causal sea insuficiente, se atribuirán entonces a ciertos factores cognitivos como las creencias o los deseos.

De acuerdo con lo anterior, según Baron-Cohen (1991, 1993), hay emociones como la sorpresa y la perplejidad que son propiamente cognitivas. Es decir, cuando percibimos estas emociones expresadas en los demás, solemos atribuirlas a factores cognitivos. Así, por ejemplo, cuando alguien manifiesta una emoción tal como la sorpresa, atribuimos su contenido a la existencia de una contradicción entre lo que la persona espera y el resultado que le ha sido ofrecido por los hechos reales; es decir, la realidad esperada o posible no ha coincidido con la realidad percibida.

De acuerdo con la teoría de la mente, los autistas deberían mostrar un déficit muy notorio en el reconocimiento de las emociones cognitivas, dada las dificultades que experimentan para ponerse en el lugar del otro, para adoptar la perspectiva de los demás.
La presente investigación tiene por objeto explorar si los autistas adultos muestran un déficit específico en el reconocimiento de una emoción, la sorpresa, conceptualizada ésta como una emoción cognitiva.

\section{MÉTODO}

\section{Sujetos}

En esta investigación participaron 17 adultos autistas, diagnosticados según los criterios del DSM-IV ( $A$ American Psychiatric Association», 1994), que recibían asistencia en el Centro Nuevo Horizonte, de las Rozas (Madrid), especializado en la rehabilitación de personas afectadas por este trastorno. La «ratio» por sexos fue de 3:1 (m:f). La edad media cronológica fue de 21.35 años y la desviación típica de 4.03. El rango de variabilidad osciló entre 18 y 28 años.

La muestra de deficientes mentales estuvo igualmente constituida por 17 sujetos de ambos sexos, que recibían atención psicopedagógica en el Colegio Virgen de Lourdes de Majadahonda (Madrid) y en el Centro Nuevo Horizonte. La media de edad cronológica en este grupo fue de 21 años, y la desviación típica de 3.52. El rango de variabilidad osciló entre 9 y 23 años. Fueron emparejados con los autistas en cuanto a edad y sexo.

La edad mental verbal y no verbal obtenidas fueron prácticamente iguales en ambos grupos de personas. La edad mental verbal fue obtenida a través de la versión española del «British Picture Vocabulary Test» (BPVS; Dunn, Dunn, Whetton, y Pintile, 1982). La edad mental no verbal fue evaluada a través del "Leiter International Test» (Leiter, 1948)

Además, como criterio de inclusión, se estableció que los sujetos tuvieran 4 años de Edad verbal como mínimo, por ser la 
edad que se considera necesaria para superar los tests de percepción de emociones de Harris et al. (1989).

Los sujetos integrantes de los dos grupos clínicos no tenían asignado ningún diagnóstico añadido al de autismo o deficiencia mental y no recibían medicación psicotrópica en el momento de la realización de las pruebas. La participación de los sujetos fue debidamente autorizada por sus representantes legales y no obtuvieron ninguna gratificación por participar en las pruebas.

La muestra de la población general estuvo constituida por 17 personas de ambos sexos, estudiantes de secundaria, bachillerato y primer curso de universidad, igualados en edad cronológica y sexo con los pacientes de los grupos de autistas y deficientes mentales. La selección de estos sujetos fue aleatoria y su participación en las pruebas fue totalmente voluntaria.

En lo que se refiere a la edad cronológica, no se detectaron diferencias significativas entre los tres grupos. Tampoco se encontraron diferencias significativas entre los deficientes mentales y los autistas en lo relativo a la edad mental verbal y no verbal y a la inteligencia no verbal.

Los detalles descriptivos de las muestras aparecen recogidos en la Tabla 1.
En el grupo de la población general se especifica solamente la edad cronológica. Las restantes variables no se describen por ajustarse a la normalidad.

Criterios de inclusión y selección de la muestra

Los grupos de edad que han sido seleccionados responden a una necesidad previamente establecida en el diseño: la de comparar los resultados experimentales obtenidos en los niños autistas por el grupo de Hobson, entre otros con los hallados en los adultos autistas, en lo relativo al mismo núcleo temático en la presente investigación.

Es cierto que la evolución del autismo infantil a lo largo de la vida se manifiesta de modo muy diverso en cada uno de los pacientes. Esto quiere decir, que la expresión de la enfermedad a través de la fenomenología sintomatológica y clínica no es ni lineal ni unívoca.

No obstante, puede identificarse en numerosos pacientes autistas adultos la persistencia de un denominador común sintomático, relativamente invariante, estable y consistente.

Todos los autistas adultos seleccionados para esta investigación, satisfacían obviamente, los criterios diagnósticos

Tabla 1. Variables demográficas: Medias, desviaciones típicas y rangos de edad cronológica (EC), edad mental no verbal (EMNV) y edad mental verbal (EMV)

\begin{tabular}{|c|c|c|c|}
\hline & EC & EMNV & EMV \\
\hline \multicolumn{4}{|c|}{ Autistas $(\mathrm{N}=17)$} \\
\hline Media & 21,35 & 6,09 & 4,99 \\
\hline DT & 4,03 & 1,96 & 2,16 \\
\hline Rango & $15-28$ & $4,17-9,92$ & $4-10$ \\
\hline \multicolumn{4}{|c|}{ Deficientes mentales $(\mathrm{N}=17)$} \\
\hline Media & 21,00 & 5,35 & 5,26 \\
\hline DT & 3,52 & 0,66 & 0,80 \\
\hline Rango & $9-23$ & $4,17-6,75$ & $4,00-6,75$ \\
\hline \multicolumn{4}{|c|}{ Población general $(\mathrm{N}=17)^{*}$} \\
\hline Media & 20,9 & 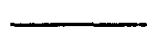 & \\
\hline DT & 2,3 & $\longrightarrow$ & - \\
\hline Rango & $14-23$ &  & \\
\hline
\end{tabular}


del DSM-IV (American Psychiatric Association, 1994). Esto supone - $y$ ha sido una condición "sine qua non» satisfecha en este diseño-, que los déficits interpersonales en la expresión y reconocimiento de las emociones, podría considerarse como un continuo en lo que se refiere a los autistas adultos, sólo sí en ellos se manifestasen de igual o parecido modo a los niños autistas esos mismos déficits.

Para este propósito, este equipo investigador determinó que los pacientes de la muestra seleccionada satisficieran idéntico núcleo sintomático al manifestado por los niños autistas, en lo que respecta a los déficits en la comunicación y en las relaciones interpersonales.

Por consiguiente, los pacientes incluidos en esta investigación eran personas adultas que satisfaciendo los criterios diagnóstícos de autismo del DSM-IV manifestaban un perfil sintomático, relativamente idéntico al observado en los niños autistas. Es decir, la muestra de autistas seleccionada estaba compuesta por personas que satisfacían los criterios de diagnóstico del autismo y que están así diagnosticadas, en la mayor parte de los casos, desde la aparición del trastorno.

A ello hay que añadir otro criterio que, aunque externo, resultó ser relevante, por cuanto que sale garante del diseño realizado. Este criterio consistió en satisfacer las recomendaciones que en 1993 el mismo Hobson sugirió a este equipo investigador: a) Utilizar grupos clínicos equiparados tanto en edad mental verbal como no verbal; b) Seleccionar tareas con estímulos emocionales de naturaleza simple y cognitiva (Hobson, 1991).

\section{Pruebas diagnósticas}

Los resultados ofrecidos en este artículo forman parte de una investigación más amplia. (García-Villamisar y PolainoLorente, 1998a y 1998b; en prensa). Aquí sólo nos referimos a las pruebas utilizadas para satisfacer los objetivos de este trabajo.

- British Picture Vocabulary Test (BPVS; Dunn, Dunn, Whetton, y Pintile, 1982). Es un test de imágenes destinado a evaluar la edad mental verbal. Ha sido ampliamente empleado en las investigaciones sobre el procesamiento de las emociones en pacientes autistas. En esta investigación se utilizó la versión española de MEPSA.

- Test de Leiter de Inteligencia no Verbal (Leiter, 1948). Es un test destinado a la evaluación de la inteligencia no verbal, que ha sido habitualmente utilizado en este tipo de investigaciones. Se utilizó la versión española de Psymtec.

\section{Material y Procedimiento}

En esta investigación se utilizaron dos series de 4 fotografías cada una, que sirvieron de muestra, y un bloque de 16 fotografías que debían de ser emparejadas con las de la muestra por los sujetos que participaron en la investigación.

Las fotografías (estímulos) expresan emociones faciales y fueron seleccionadas entre las fotografías menos ambiguas y más fiables de la colección de Ekman y Friesen (1976).

Para este propósito, se realizó una reproducción fotográfica de las fotografías originales, al tamaño de $24 \times 16$ $\mathrm{cm}$, y fueron enmarcadas en un cuadro de forma oval, de manera que no pudieran reconocerse ni las orejas ni el pelo.

En esta investigación se agruparon los estímulos emocionales en dos bloques: emociones simples (alegría, tristeza y miedo) y emociones cognitivas (sorpresa). 


\section{Prueba de screening}

A fin de verificar que los sujetos comprendían y eran capaces de seguir las instrucciones del test se aplicó, de acuerdo con Hobson, Ouston y Lee (1988), una prueba de "screening" que constaba de las siguientes tareas:

1) Seleccionar tres fotografías de un total de seis de diferentes personas de la muestra, que expresaban alegría y emparejarlas con las fotografías correspondientes de la muestra que mostraban idéntico sentimiento (alegría).

2) Seleccionar tres fotografías de un total de seis, de la misma persona la muestra que expresan diversas poses emocionales y emparejarlas con la fotografía de la muestra (pose neutral).

Sólo se seleccionaron a aquellas personas que no cometieron errores en el único ensayo realizado.

\section{Tarea experimental: Test de} reconocimiento de las emociones

La tarea experimental consistió en un test de reconocimiento de las emociones, según el procedimiento de emparejamiento con la muestra, que constaba de los dos componentes siguientes:

a) Estímulos de la muestra: Los estímulos de la muestra consistieron en 4 fotografías de una misma persona (cuya foto no aparecía entre las fotografías a clasificar), dispuestas en semicírculo, en las que se expresan diversos estados emocionales como alegría, tristeza, sorpresa y miedo. Estas fotografías sirvieron de modelo para la comparación.

b) Estímulos de la tarea: Los estímulos de la tarea consistieron en 16 fotografías de diversas personas, que manifestaban diversos estados emocionales como alegría, tristeza, sorpresa y miedo.
Se dispusieron las fotografías de forma aleatoria en series de 4 en 4 , formando una matriz cuadrada $2 \times 2$, sobre una mesa de $1 \mathrm{~m}^{2}$ de superficie y se solicitó a cada probando que situase la foto de la matriz con la que se correspondía en la base de cada una de las fotografías de la muestra.

Es decir, la tarea consistió en emparejar las fotografías, según los estados emocionales que muestran las fotografías de los diversos sujetos de la tarea, respecto de las emociones expresadas por el mismo sujeto en las fotografías de la muestra.

\section{Estímulos invertidos}

En la tarea experimental se ofreció una segunda versión, idéntica a la descrita hasta aquí, sólo que las fotografías estaban dispuestas de forma invertida.

Instrucciones: El experimentador nombraba las emociones expresadas en la muestra y pedía a cada sujeto que emparejase cada fotografía de la tarea con la correspondiente de la muestra.

Diseño: Se empleó un diseño factorial mixto con dos factores intrasujeto: Tipo de estímulos emocionales (emociones simples vs cognitivas) y modalidad de presentación de estímulos (directa vs invertida) y un factor intersujeto (diagnóstico clínico). Las variables dependientes fueron: precisión de respuesta (número de aciertos) y tiempo de respuesta (tiempo invertido en la realización de la tarea)

\section{Hipótesis}

1. Se estima que los autistas emitirán menos respuestas acertadas en la modalidad de presentación directa de los estímulos relativos a la sorpresa que los deficientes mentales y las personas de la 
población general aunque no sucederá en la presentación inversa de esos mismos estímulos.

2. Se estima que los autistas invertirán más tiempo en la resolución de tareas relativas a la situación estimular de sorpresa, en la modalidad de presentación directa, que los deficientes mentales y las personas de la población general, , pero no sucederá así en la presentación inversa de estos mismos estímulos.

\section{RESULTADOS}

1. Respecto a la precisión de respuesta (número de aciertos alcanzados) en las diversas pruebas de reconocimiento de emociones simples y cognitivas

La Tabla 2 contiene las medias y las desviaciones típicas de las puntuaciones logradas en las tareas de reconocimiento de las emociones simples y cognitivas (sorpresa).

Se realizó un análisis de varianza correspondiente a un diseño factorial mixto $\mathrm{A} \times \mathrm{B} \times \mathrm{C}$, con medidas repetidas en $\mathrm{B}$ y $\mathrm{C}$, siendo

A: Categorías diagnósticas. 3 niveles: autistas, deficientes mentales y sujetos de población general.
B: Tipo de estímulos emocionales. 2 niveles: emociones simples y emociones cognitivas

C: Modalidad de presentación de los estímulos. 2 niveles: normal e invertida.

Los efectos obtenidos fueron significativos respecto de las categorías diagnósticas $\left(\mathrm{F}_{(2,47)}=39,21 ; \mathrm{p}=0.000\right)$. El análisis de los principales efectos encontrados en esta variable reveló que no había diferencias significativas entre los autistas y los deficientes mentales; por contra, sí se apreciaron diferencias significativas tanto entre la población general y los deficientes mentales $\left(F_{(1,47)}=41,97 ; p\right.$ $=0.000$ ), como entre la población general $y$ los autistas $\left(F_{(1,47)}=71,31 ; p=0.000\right)$, tal como cabía esperar.

2. Respecto del tiempo invertido en la realización de las diversas pruebas de reconocimiento de emociones simples y cognitivas.

En la Tabla 3 se exponen las medias y desviaciones típicas de los tiempos invertidos (expresado en segundos) por los diversos grupos en la realización de las pruebas.

Se realizó un análisis de varianza correspondiente a un diseño factorial $\mathrm{A} \times \mathrm{B} \times \mathrm{C}$, con medidas repetidas en $\mathrm{B}$ y $\mathrm{C}$, siendo

Tabla 2. Medias y Desviaciones típicas de los resultados alcanzados en las pruebas de reconocimientos de emociones simples y cognitivas, tanto en la modalidad de presentación directa (PD) de los estímulos, como invertida (PI)

\begin{tabular}{|c|c|c|c|c|}
\hline \multirow{3}{*}{$\begin{array}{c}\text { Categorías } \\
\text { diagnósticas }\end{array}$} & \multirow{2}{*}{\multicolumn{2}{|c|}{$\begin{array}{l}\text { Tipo de } \\
\text { Emociones simples }\end{array}$}} & \multirow{2}{*}{\multicolumn{2}{|c|}{$\begin{array}{l}\text { cionales } \\
\text { Emociones cognitivas }\end{array}$}} \\
\hline & & & & \\
\hline & PD & PI & $\mathrm{PD}$ & PI \\
\hline \multicolumn{5}{|l|}{ AUTISTAS } \\
\hline $\mathbf{x}$ & 1,59 & 1,59 & 1,89 & 1,47 \\
\hline$\sigma$ & 0,90 & 0,89 & 1,36 & 1,37 \\
\hline \multicolumn{5}{|c|}{ DEFICIENTES MENTALES } \\
\hline$x$ & 2,09 & 2,00 & 2,06 & 2,00 \\
\hline$\sigma$ & 0,85 & 0,93 & 1,43 & 1,17 \\
\hline \multicolumn{5}{|c|}{ POBLACION GENERAL } \\
\hline $\mathbf{x}$ & 3,92 & 3,96 & 3,71 & 3,29 \\
\hline $\boldsymbol{\sigma}$ & 0,22 & 0,11 & 0,47 & 0,17 \\
\hline
\end{tabular}


Tabla 3. Medias y Desviaciones típicas del tiempo empleado (expresado en segundos) en la resolución de las tareas de reconocimiento de emociones simples y cognitivas, en las modalidades de presentación directa (PD) de los estímulos e invertida (PI)

\begin{tabular}{|c|c|c|c|c|}
\hline \multirow{3}{*}{$\begin{array}{c}\text { Categorías } \\
\text { diagnósticas }\end{array}$} & \multicolumn{4}{|c|}{ Tipo de estímulos emocionales } \\
\hline & \multicolumn{2}{|c|}{ Emociones simples } & \multicolumn{2}{|c|}{ Emociones cognitivas } \\
\hline & $\mathrm{PD}$ & PI & PD & PI \\
\hline \multicolumn{5}{|l|}{ AUTISTAS } \\
\hline $\mathbf{x}$ & 39,04 & 34,88 & 40,48 & 35,00 \\
\hline$\sigma$ & 13,33 & 13,95 & 15,38 & 7,61 \\
\hline \multicolumn{5}{|c|}{ DEFICIENTES MENTALES } \\
\hline $\mathbf{x}$ & 15,92 & 14,10 & 19,06 & 17,76 \\
\hline 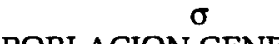 & 12,22 & 2,65 & 20,02 & 22,38 \\
\hline \multicolumn{5}{|c|}{ POBLACION GENERAL } \\
\hline $\mathbf{x}$ & 0,80 & 1,35 & 1,82 & 3,12 \\
\hline$\sigma$ & 0,47 & 0,51 & 0,8 & 0,93 \\
\hline
\end{tabular}

A: Categorías diagnósticas: 3 niveles: autistas, deficientes mentales y sujetos de población general.

B: Tipo de estímulos emocionales. 2 niveles: emociones simples y emociones cognitivas

C: Modalidad según la presentación de estímulos. 2 niveles: normal e invertida.

El criterio de respuesta fue el tiempo empleado en la realización de las diversas pruebas (expresado en segundos).

Los resultados obtenidos fueron significativos, en lo relativo a las diversas categorías diagnósticas $\left(\mathrm{F}_{(2,47)}=68,42\right.$; $\mathrm{p}<0,0001$ y al Tipo de estímulos emocionales $\left(F_{(1,47)}=189,16 ; p=0,000\right)$ e interacción Tipo de estímulos emocionales por categorías de diagnóstico $\left(F_{(2,47)}=\right.$ 63,$82 ; p=0,000$ ). Los contrastes planificados de la interacción pusieron de manifiesto la presencia de diferencias significativas entre los autistas en cuanto al tiempo utilizado $\left(\mathrm{F}_{(1,47)}=22,29 ; \mathrm{p}=\right.$ $0,000)$, siendo más rápidos para reconocer los estímulos emocionales simples (emplearon un tiempo promedio de 36,96 segundos), que en el reconocimiento de estímulos emocionales de tipo cognitivo (tiempo promedio de 37,71 segundos).

\section{DISCUSIÓN}

El objetivo de este trabajo era probar que los adultos autistas tenían ciertas dificultades para reconocer los estímulos emocionales, particularmente, cuando estos eran de naturaleza cognitiva. Con anterioridad, otros investigadores habían demostrado la presencia de este déficit en niños (Baron-Cohen, Spitz y Cross, 1993), pero se desconocía si esta dificultad se apreciaba también en personas adultas afectadas por el trastorno autista.

Se partió del supuesto de que los autistas parecían disponer de menos dificultades para percibir las emociones simples (alegría, tristeza, miedo, etc.) que las emociones cognitivas (sorpresa). De acuerdo con las investigaciones previamente realizadas por Baron-Cohen et al., (1985 y 1993), y Perner et al. (1989), los autistas tienen más dificultades para percibir las emociones que aparecen asociadas con las creencias (emociones cognitivas) que aquellas otras que aparecen asociadas con las situaciones (emociones simples). Sin embargo, está por dilucidar si este déficit se mantiene a lo largo del desarrollo.

Los resultados obtenidos en esta investigación demuestran que no aparecen diferencias significativas entre los autis- 
tas adultos y los deficientes mentales, tanto en el reconocimiento de las emociones simples como de las emociones cognitivas. Sin embargo, los sujetos normales obtuvieron un rendimiento mucho más aceptable en estas pruebas que los deficientes mentales y los autistas, tal como se hipotetiz6́.

Por lo que se refiere al tiempo empleado en la resolución de las tareas, los resultados de esta investigación son coincidentes con los ofrecidos por BaronCohen et al. (1993) con respecto a los niños, en el sentido de que los autistas adultos, al igual que los niños autistas, invirtieron más tiempo para resolver las tareas implicadas en el reconocimiento de emociones cognitivas que en las de las emociones simples.

Dicho en otras palabras, los autistas adultos son tan eficaces a la hora de resolver las tareas de reconocimiento de las emociones simples como las cognitivas. Sin embargo, utilizan más tiempo en la resolución de las tareas cognitivas que en las tareas relativas a las emociones simples. Esta mayor latencia de respuesta en las tareas de reconocimiento de emociones cognitivas puede ser atribuida al déficit metarrepresentacional. Tal como han demostrado diversos estudios de la teoría de la mente (Baron-Cohen, 1993), los autistas encuentran muy difícil reconocer las emociones cognitivas, pues fallan a la hora de hacer las segundas representaciones que exige la comprensión de las emociones cognitivas.

Estos resultados ofrecen un cierto apoyo indirecto a la "teoría de la mente", dado que los autistas adultos parecen mostrar ciertas dificultadas en la resolución de tareas que, desde luego, exigen el concurso de las facultades cognitivas. Por otra parte, esta investigación prueba la existencia de un cierto isomorfismo continuista entre los niños y los adultos autistas en cuanto a que los adultos autistas, al igual que los niños, tienen una mayor dificultad para reconocer las emociones de tipo cognitivo.

En conclusión, los autistas adultos son igual de precisos tanto en el reconocimiento de emociones simples como cognitivas. Sin embargo, son más lentos a la hora de resolver las tareas cuyos contenidos se refieren a las emociones cognitivas. Estos resultados parecen producirse con independencia de que los estímulos se presenten de forma directa o invertida.

\section{REFERENCIAS BIBLIOGRÁFICAS}

American Psychiatric Association. (1994). Diagnostic and statistical manual of mental Disorders. Washington.

Baron-Cohen, S. (1991) Do people with autism understand what causes emotion? Child Development, 62, 385-395.

Baron-Cohen, S. (1993). From attention-goal psychology to belief-desire psychology: the development of a theory of mind, and its dysfunction. En S. Baron-Cohen, H. TagerFlusberg y D. Cohen, Understanding other minds: perspectives from autism. Oxford University Press.

Baron-Cohen, S., Lesie, A.M., y Frith, U. (1985). Does the autistic child have a theory of mind? Cognition, 21, 37-46.

Baron-Cohen, S., Spitz, A., y Cross, P. (1993). Do children with autism recognise surprise? A research note. Cognition and Emotion, 7, 507-516.

Baron-Cohen, S., Tager-Flusberg, H., y Cohen, D. (1993). Understanding other minds: perspectives from autism. Oxford University Press.

Borke, H. (1971). Interpersonal perception of young children: Egocentrism or empathy? Developmental Psychology, 5, 263-269.

Duun, L.M., Duun, L.M., y Whetton, C. (1982). British Picture Vocabulary Scale. (Versión española: TEA).

Ekman, P., y Friesen, W.V. (1976). Unmasking the face. $A$ guide to recognizing emotions from facial cues. Englewood Cliffs, N.Y: Prentice-Hall.

García-Villamisar, D., y Polaino-Lorente, A. (1998a). Attribution causale de certains 
états emotionnels manifestés par les jeunes autistes: Une analyse d'exploration. Actas del V Congreso Internacional de AutismoEuropa. Edit. Aula Abierta. Fundación ONCE. Madrid.

García-Villamisar, D., y Polaino-Lorente, A. (1998b, en prensa). Las emociones y el comportamiento autista. Promolibro.

García-Villamisar, D., y Polaino-Lorente, A. (1998c). Análisis diferencial de la percepción de emociones entre autistas, deficientes mentales y población general. Análisis y Modificación de Conducta, 24, 94, 195216.

Happé, F. (1994) Autism. An introduction to psychological theory. UCL Press: London.

Harris, P. (1989). Children and emotion. Oxford: Blackwell.

Harris, P. (1993). Understanding emotion. En M. Lewis y J. Haviland (Eds.), Handbook of emotions. Guilford Press.

Harris, P. (1994). The child's understanding of emotion: developmental change and the family environment. Journal of Child Psychology and Psychiatry, 35, 3-28.

Harris, P.L. (1989). Children and Emotion. Oxford: Basil Blakwell.

Hobson, P. (1991). Methodological issues for experiments on autistic individual's perception and understanding of emotion. Journal of Child Psychology and Psychiatry, 32, 1135-1158.

Hobson, P. (1993). Autism and the development of mind. Lawrence Erlbaum Associates, Publishers.

Hobson, P., Ouston, J., y Lee, T. (1988).
What's in a face? The case of autism. British Journal of Psychology, 79, 441-453.

Kanner, L. (1943). Autistic disturbances of affective contact. Nervous Child, 2, 217-230.

Leiter, R.G, (1948). Leiter International Performance Scale. Chicago: Stoelting.

Leslie, A.M. (1988). Some implications of pretence for mechanisms underlyn the child's theory of mind. En J. Astington, P. Harris y D. Olson (Eds.), Developing theories of mind. Cambridge: MIT press.

Leslie, A.M. (1993). What autism teaches us about metarepresentation. En S. BaronCohen, H. Tager-Flusberg y D. Cohen (1993). Understanding other minds: perspectives from autism. Oxford University Press.

Perner, J. (1991). Understanding the representational mind. Cambridge: MIT press.

Trabasso, R., Stein, N.L., y Johnson, L. (1981). Children's knowledge of events: A causal analysis of story structure. En G. Bower (Ed.), Learning and motivation (Vol. 15, pp. 237-282). NY: Academic Press.

Wellman, H., y Woolley, J. (1990). From simple desires to ordinary beliefs: The early development of everyday psychology. Cognition, 35, 245-275.

Wellman, H. (1990). Children's theories of mind. Cambridge: MIT press.

Wellman, H., y Bartsch, K. (1988)., young children's reasoning about beliefs. Cognition, 30, 239-277.

Yuill, N. (1984)., young children's coordination of motive and outcome in judgments of satisfaction and morality. British Journal of Development Psychology, 2, 73-81. 